\title{
Erratum: Characteristics of quantum-classical correspondence for two interacting spins [Phys. Rev. A 63, 052103 (2001)]
}

\author{
J. Emerson and L. E. Ballentine
}

(Published 3 July 2001)

DOI: 10.1103/PhysRevA.64.029901

PACS number(s): 03.65.Sq, 05.45.Mt, 03.65.Ta, 99.10.+g

Unfortunately an alteration in proof was incorrectly implemented, leading to an error in Eq. (45). The tildes ( $\sim)$ should be removed from the symbols in the numerator of Eq. (45). These numerators should denote the actual magnitudes of the quantum-classical differences between the angular momentum components, not the rescaled values (as would be indicated by the tildes). The equation should read as follows:

$$
\frac{\delta L_{z}\left(t^{*}\right)}{\sqrt{l(l+1)}} \leqslant \frac{\delta L_{z}^{\max }}{\sqrt{l(l+1)}} \sim O(1 / l) .
$$

